\title{
Smoking Onset Year
}

National Cancer Institute

\section{Source}

National Cancer Institute. Smoking Onset Year. NCI Thesaurus. Code C156423.

The year in which an individual began smoking. 